\title{
Prevalence of sexual dysfunction in women with systemic lupus erythematosus and its related factors
}

\author{
Zahra Behboodi Moghadam ${ }^{1}$, Elham Rezaei ${ }^{2,3}$, Seyedeh Tahereh Faezi ${ }^{4}$, Armin Zareian ${ }^{5}$, \\ Fatima Muhammad Ibrahim², Maryam Mohammad Ibrahim² \\ ${ }^{1}$ Reproductive Health, Faculty of Nursing and Midwifery, Tehran University of Medical Sciences, Iran \\ ${ }^{2}$ Candidate of Reproductive Health, School of Nursing and Midwifery, Tehran University of Medical Sciences, Iran \\ ${ }^{3}$ School of Nursing and Midwifery, Urmia University of Medical Sciences, Iran \\ ${ }^{4}$ Rheumatology Research Center, Tehran University of Medical Sciences, Iran \\ ${ }^{5}$ Public Health Department, Nursing Faculty, Aja University of Medical Science, Tehran, Iran
}

\begin{abstract}
Objectives: Systemic lupus erythematosus (SLE) affects all body organs. This disease has marked physiological, mental, and cognitive effects on the patient and results in sexual dysfunction, depression, and decreased self-confidence. This study was conducted to compare sexual function, depression, anxiety, stress, and the related factors in women suffering from SLE.

Material and methods: In this cross-sectional study, 340 women (170 with SLE and 170 healthy) aged 18-49 years were investigated. Convenience sampling was used to select the participants. In this study, a demographic questionnaire, the Depression, Anxiety, and Stress Scales - 21 Items (DASS-21), and the Female Sexual Function Index (FSFI) were applied. Data were analyzed with SPSS 16 using Spearman's correlation, $\chi^{2}$, Mann-Whitney, covariance, and multiple regression statistics; $p$-values less than 0.05 were considered significant.

Results: The results showed a significant difference in sexual function between the SLE and healthy groups. According to covariance analysis, the difference remained significant after controlling for confounding factors (stress, depression, anxiety). Moreover, the effect of SLE on the sexual function had a significant correlation with age, life status, number and age of children, economic status, menstruation, duration of marriage, age at diagnosis, disease duration and activity, stress, anxiety, and depression. The results of multiple regression analysis showed that age, disease activity, depression, and life status had the highest correlation with sexual dysfunction in women with SLE. Conclusions: The results of this study indicated that more attention should be paid to the sexual health of women suffering from SLE as a neglected aspect of their treatment, which requires an interdisciplinary approach to shift the care of these patients from a biomedical model to a biopsychosocial model.
\end{abstract}

Key words: systemic lupus erythematosus, sexual dysfunction, women.

\section{Introduction}

Systemic lupus erythematosus (SLE) is a chronic, complicated, autoimmune disease with cumulative involvement of different organs. Due to unknown reasons, SLE is now 10 times more prevalent in developed countries than it was 50 years ago. It is believed that the prevalence of SLE is much lower in Africa and Asia in comparison with Europe (western countries). However, in industrialized Western countries, the odds of SLE are 2-4 times higher in people of African and Asian origin as compared to European people [1-3]. In a study by Jones et al. [4], the overall incidence and prevalence of SLE were 4.9-5.5 and 72.8-97 in 100,000 in England respectively. There are 322,000-1,500,000 SLE patients in

Address for correspondence:

Elham Rezaei, Department of Reproductive Health, Faculty of Nursing and Midwifery, Tehran University of Medical Sciences, Nosrat St., Tohid Sq., 1419733171 Tehran, Iran, e-mail: rezai520@yahoo.com

Submitted: 17.10.2018; Accepted: 2.01.2019 
the United States. Women are affected nine times more than men are $(6-10: 1)$, and the disease is more common in women of reproductive age $[1,2]$.

As a country located in the Middle East, Iran is a good candidate for lupus research considering the similarity of its population to the European ethnicity. The results of a review study by Akbarian et al. [5] in 2280 SLE patients in Iran showed that women were affected nine times more than men were. The mean age at presentation was 24 years, and the age range of patients was $15-45$ years [5].

According to a report of the Rheumatology Research Center of Tehran University of Medical Sciences, SLE has the highest morbidity among autoimmune diseases (7\%) and is considered the mother of autoimmune diseases. According to a report of the SLE Unit of Shariati Hospital affiliated with Tehran University of Medical Sciences as a lupus referral center, the prevalence of this disease is 40 in 100,000 population in Iran [3, 5].

Systemic lupus erythematosus affects all organs and has marked physiological, mental, and cognitive impacts on the patients. It results in sexual and physical dysfunction, depression, and decreased self-confidence. In increases stress and depression by $5-80 \%$ in female patients [6].

A study by Morales et al. [7] in Spain showed that persistent pain and fatigue related to SLE could hinder physical and emotional aspects of a sexual relationship. The authors found that the rate of avoiding a sexual relationship was higher (50\%) and the rates of desire, arousal, lubrication, and orgasm were lower in patients with SLE [7]. Sexual dysfunction is associated with physical complaints, obsessive-compulsive symptoms, interpersonal sensitivity, psychoticism, decreased vitality, and impaired social function and mental health [8, 9].

In a Chinese study in 2015, Shen et al. [10] found that $64 \%$ of the SLE patients had impaired sexual function. The authors emphasized that age, marital status, depression, and body image disturbances were the strongest predictors of impaired sexual function in SLE patients.

Moreover, Knight et al. [11] and McElhone et al. [12] reported that fatigue was a global SLE symptom resulting in marital and family problems through causing sexual, physical, mental, and psychological dysfunction. They found that SLE decreased all aspects (physical, sexual, mental, and social) of the quality of life of female patients [8, 11, 12].

The objective of United Nations Population Fund is to provide global access to health, sexual rights, and reproduction, and all countries are committed to promotion of women's health (according to the 2030 agenda for Sustainable Development Goals and the WHO strategy on women's health and well-being 2021) [13]. In this regard, considering the importance of chronic diseases due to their high morbidity, SLE is an important autoimmune disease with a high morbidity rate that warrants special attention.

There are no accurate data on sexual dysfunction in Iranian patients with SLE, and this aspect of health is not evaluated in Iranian women suffering from chronic diseases due to cultural issues. Therefore, we conducted a study to evaluate sexual dysfunction in female SLE patients.

\section{Material and methods}

This cross-sectional study was part of a PhD thesis on reproductive health. The study was conducted in the Rheumatology and Women's Clinics of Shariati Hospital (referral centers for rheumatology and women's diseases), Tehran University of Medical Sciences, Iran from August 2017 to February 2018. The study population comprised 340 women (170 SLE patients and 170 healthy controls). Convenience sampling was used to select the participants. Three questionnaires were used in this study: a demographic questionnaire, the Depression, Anxiety, and Stress Scales-21 Items (DASS-21), and the standardized Persian version of the Female Sexual Function Index (FSFI).

After obtaining the approval of the Ethics Committee of Tehran University of Medical Sciences (ethics code: 34933 dated September 3, 2017), the researcher and research assistant attended the Rheumatology Clinic (for sampling women with SLE) and Women's Clinic (to sample control subjects) in working hours. They introduced themselves to the authorities and women attending these clinics and explained the objective of the study. The participants were informed that their information would be anonymous and confidential and that they could withdraw from the study at any time. Informed consent was then obtained from all participants before initiation of the study. Eligible women (age 18-49 years, married, Iranian, having no known physical or mental diseases affecting a sexual relationship, not using hormonal drugs or drugs affecting a sexual relationship) were asked to complete the questionnaires in a calm and quiet environment in the presence of a researcher or research assistant. In addition, eligible women completed the DASS-21 for evaluation of depression before entering the study, and cases with major depressive disorder were referred to the psychiatrist and mild and moderate cases were included in the study.

The demographic questionnaire was used to collect the data of age, occupation, education, life status, number and age of children, economic status, menstruation status, and duration of marriage. The Systemic Lupus Erythematosus Disease Activity Index (SLEDAI) was applied to assess age at diagnosis, duration, and activity of SLE. 
The FSFI was used to assess sexual performance, dysfunction, and satisfaction. This questionnaire has 19 five-choice questions to assess the domains of sexual desire (questions 1 and 2), arousal (questions 3-6), orgasm (questions 11-13), vaginal lubrication (questions 7-10), sexual satisfaction (questions 14-16), and pain or discomfort following vaginal penetration (questions 17-19). According to the instructions provided by the designer, to calculate the score of each individual domain, the scores of the individual questions comprising the domain are added and multiplied by the domain factor (0.6 for desire, 0.3 for arousal, 0.3 for lubrication, 0.3 for orgasm, 0.4 for satisfaction, and 0.4 for pain). The scores of desire, satisfaction, and other domains are in the ranges $1.2-6,0.8-6$, and $0-6$, respectively. The scores of six domains are added to obtain the full scale score. A higher score indicates better sexual performance. In this questionnaire, the cut points of desire, arousal, lubrication, orgasm, pain, satisfaction, and the whole scale are 2.1, 2.8, 2.8, 2.6, 3, 3, and 26.55, respectively. A score $\leq 26.55$ indicates sexual dysfunction and a score above 26.55 indicates normal function. The reliability and validity of this scale have been confirmed in several studies in Iran and other countries [10, 14].

The Depression, Anxiety, and Stress Scales - 21 Items (DASS-21) was used to assess depression, anxiety, and stress (emotional reactions). It was first developed by Loibound in 1995 and has answer choices as not at all, to some degree, to a considerable degree, and very much. Each question is scored from 0 to 3 . One study showed a high correlation between the DASS-21 and Beck's Depression Inventory (BDI). Crawford and Henry [15] compared the DASS-21 with two other measures of depression and anxiety and a measure of positive and negative affectivity. They concluded that the best condition for the DASS-21 was when all three factors (stress, anxiety, and depression) were considered in it. The reliability and validity of this questionnaire have been confirmed in Iranian studies [16].

SPSS version 16 was used for data analysis. The Kolmogorov-Smirnov test was applied to assess data normality. Descriptive statistics were used for frequency distribution, measures of central tendency, and tables. Spearman's correlation coefficient was used to investigate correlations between variables, and $\chi^{2}$, Mann-Whitney, and covariance tests were applied to compare the mean values of variables between the two groups in terms of demographic and other factors. A multiple regression model was applied to predict the status of dependent variables; $p$-values less than 0.05 were considered significant.

\section{Results}

In this study, 170 women with SLE and 170 healthy women were examined. The mean age of the patients and controls was $37.64( \pm 7.96)$ and $33.77( \pm 6.64)$ years, respectively. The disease was inactive in $68.3 \%$ and active in $32.7 \%$ of the patients (mild and moderate in $26.5 \%$ and severe in $8.2 \%$ ). According to the demographic characteristics of the women presented in Table I, $48.2 \%$ had high school education, $88.2 \%$ were housewives, and $68.2 \%$ were of the middle economic class. The mean duration of marriage was 15.07 ( \pm 9.8$)$ years. There was no significant difference in demographic characteristics between the two groups according to the Man-Whitney and $\chi^{2}$ tests.

According to Table II and Figure 1, there were significant differences in all domains of sexual function except for pain between cases and controls. The results of covariance analysis after controlling for the effect of confounders (depression, anxiety, and stress) showed a significant difference in the sexual performance and the domains of desire, arousal, and orgasm between cases and controls.

Sexual dysfunction, stress, anxiety, and depression were seen in $85.9 \%, 62.9 \%, 63.5 \%$, and $85.9 \%$ of the women with SLE and 26.5\%, 29.5\%, 27.6\% and $31.8 \%$ of the control subjects, respectively. Moreover, the mean score of sexual function was lower in SLE women compared to controls $(20.79 \pm 6.41$ vs. $24.19 \pm 7.91)$.

According to the Spearman correlation analysis, the effect of SLE on sexual performance had a significant relationship with age, life status, number and age of children, economic status, menstruation, duration of marriage, age at diagnosis, duration and activity of SLE, anxiety, stress, and depression. The results are presented in Table III. Moreover, according to covariance analysis, the variables of number of children, duration of SLE, and anxiety had confounding effects on the relationship between SLE and sexual function while other variables did not have a confounding effect.

Stepwise regression analysis was applied to confirm the variables that had the highest correlation with sexual dysfunction in women suffering from SLE. The results showed that age, SLE activity, depression, and life status had the highest significant correlation with sexual dysfunction (Table IV) in descending order. Age alone, age and SLE activity, age and SLE activity and depression, and age and SLE activity and depression and life status predicted $17 \%\left(r_{s}: 0.171 ; p<0.001\right), 26 \%\left(r_{s}: 0.263\right.$; $p<0.001), 34 \%\left(r_{s}: 0.343 ; p<0.001\right)$, and 38\% $\left(r_{s}: 0.382\right.$; $p<0.001)$ of the changes of sexual performance in SLE women respectively.

\section{Discussion}

The correlation of sexual dysfunction with demographic variables, disease characteristics, depression, anxiety, and stress in Iranian women with SLE indicates that these patients are more prone to sexual dysfunction than healthy 
Table I. Sociodemographic variables and disease characteristics of patients with systemic lupus erythematosus and healthy controls

\begin{tabular}{|c|c|c|c|}
\hline \multirow[t]{2}{*}{ Characteristic } & SLE & Controls & $\chi^{2}$ or $Z$-value* \\
\hline & $(n=170)$ & $(n=170)$ & \\
\hline Age, mean $\pm S D$ years & $37.64 \pm 7.96$ & $33.77 \pm 6.64$ & $Z: 4.54 ; 0.07$ \\
\hline Life status, $n(\%)$ & & & $\chi^{2}: 1.02 ; 0.31$ \\
\hline With a partner & $164(96.5)$ & 169 (99.4) & \\
\hline Without a partner & $6(3.5)$ & $1(0.6)$ & \\
\hline Educational level, $n$ (\%) & & & $\chi^{2}: 15.87 ; 0.09$ \\
\hline Primary education & $18(10.5)$ & $12(7.06)$ & \\
\hline Secondary education & $25(14.7)$ & $16(9.41)$ & \\
\hline Higher education & $83(48.9)$ & $63(37.06)$ & \\
\hline University & $44(25.9)$ & $79(46.47)$ & \\
\hline Job, $n(\%)$ & & & $\chi^{2}: 26.15 ; 0.06$ \\
\hline Housewife & $150(88.2)$ & $110(64.7)$ & \\
\hline Employed & $20(11.8)$ & $60(35.3)$ & \\
\hline Child number, mean \pm SD & $1.41 \pm 1.06$ & $1.08 \pm 1.12$ & $Z:-3.05 ; 0.07$ \\
\hline Child age, mean $\pm S D$ & $11.35 \pm 9.9$ & $6.31 \pm 8.84$ & $Z:-5.46 ; 0.06$ \\
\hline Economic status, $n(\%)$ & & & $\chi^{2}: 3.16 ; 0.20$ \\
\hline Good & $24(14.1)$ & $20(11.8)$ & \\
\hline Moderate & $116(68.2)$ & $130(76.5)$ & \\
\hline $\mathrm{Bad}$ & $30(17.6)$ & 20 (11.8) & \\
\hline Menstrual status, $n(\%)$ & & $\chi^{2}: 16.19 ; 0.09$ & \\
\hline Normal & $112(65.9)$ & $144(84.7)$ & \\
\hline Abnormal & $58(34.1)$ & $26(15.3)$ & \\
\hline Marriage duration, mean $\pm S D$ & $15.07 \pm 9.8$ & $11.24 \pm 7.66$ & $Z:-3.47 ; 0.11$ \\
\hline Diagnostic age, mean $\pm S D$ & $27.95 \pm 7.58$ & - & - \\
\hline Disease duration, mean $\pm \mathrm{SD}$ & $9.35 \pm 7.31$ & - & - \\
\hline SLEDAI, $n(\%)$ & & - & - \\
\hline No activity & $111(65.3)$ & & \\
\hline Mild or moderate & $45(26.5)$ & & \\
\hline Severe & $14(8.2)$ & & \\
\hline
\end{tabular}

SLE - systemic lupus erythematosus; SLEDAI - Systemic Lupus Erythematosus Disease Activity Index; * using a Mann-Whitney or a $\chi^{2}$ test.

women are. Moreover, they experience higher levels of depression, anxiety, and stress. The present study is the most recent and the only study investigating these parameters in Iranian female SLE patients. The findings of this study should be considered in SLE clinics because sexual health is a forgotten aspect of treatment in these patients. According to study by Ho et al. [17], $86 \%$ of specialists and $92 \%$ of care givers of SLE patients stated that they had never addressed sexual factors in the care programs.

According to different studies, sexual disorders in SLE patients are multifactorial, but can be assessed through evaluation of physical symptoms [18, 19], in- cluding pain, fatigue, joint stiffness, and psychological reactions to chronic diseases such as depression and anxiety [20, 21].

In line with our findings, in a study by Shen et al. [10], $55 \%$ of SLE patients reported impaired sexual relationships with a partner and $64 \%$ reported impaired sexual function. Moreover, according to the results of this study, body image disturbance, education, disease activity, and depression were the most significant causes of sexual dysfunction [10]. In a study by Panush et al. [22] 20\% of SLE patients reported that the disease impaired their relationship with their spouses and the anxiety resulting 
Table II. Sexual status, stress, anxiety, and depression in systemic lupus erythematosus patients in Iran

\begin{tabular}{|c|c|c|c|c|c|c|}
\hline & \multirow{2}{*}{$\begin{array}{c}\text { SLE } \\
(n=170)\end{array}$} & \multirow{2}{*}{$\begin{array}{l}\text { Control } \\
(n=170)\end{array}$} & \multicolumn{2}{|c|}{ Mann-Whitney test } & \multicolumn{2}{|c|}{ ANCOVA } \\
\hline & & & $Z$ & $p$ & $F$ & $p$ \\
\hline FSFI (2-36) & $20.79 \pm 6.41$ & $24.19 \pm 7.91$ & -7.11 & $<0.001$ & 9.02 & 0.003 \\
\hline$\geq 26.55$ & $146(85.9)$ & $45(26.5)$ & & & & \\
\hline$<26.55$ & $24(14.1)$ & $125(73.5)$ & & & & \\
\hline 1. Desire (1-5) & $3.07 \pm 1.16$ & $3.52 \pm 1.22$ & -7.06 & $<0.001$ & 31.03 & $<0.001$ \\
\hline 2. Arousal (0-5) & $3.07 \pm 1.37$ & $3.50 \pm 1.49$ & -6.27 & $<0.001$ & 17.16 & $<0.001$ \\
\hline 3. Lubrication (0-5) & $3.99 \pm 1.50$ & $4.48 \pm 4.10$ & -4.71 & $<0.001$ & 2.77 & 0.09 \\
\hline 4. Orgasm (0-5) & $3.85 \pm 1.50$ & $4.09 \pm 1.63$ & -4.74 & $<0.001$ & 4.16 & 0.04 \\
\hline 5. Satisfaction (0-5 or $1-5)$ & $4.29 \pm 1.42$ & $4.47 \pm 1.46$ & -4.34 & $<0.001$ & 3.38 & 0.06 \\
\hline 6. Pain (1-5) & $2.56 \pm 1.18$ & $2.87 \pm 4.79$ & -1.94 & 0.052 & 3.25 & 0.07 \\
\hline DASS (0-126) & $50.95 \pm 30.76$ & $37.85 \pm 25.50$ & -3.89 & $<0.001$ & & \\
\hline 1. Stress $(0-42)$ & $21.08 \pm 10.68$ & $16.15 \pm 9.79$ & -4.29 & $<0.001$ & 0.79 & \\
\hline Normal (0-10) & $63(37.1)$ & $120(70.5)$ & & & & \\
\hline \multicolumn{7}{|l|}{ Mild (11-18) } \\
\hline \multicolumn{7}{|l|}{ Moderate (19-26 } \\
\hline \multicolumn{7}{|l|}{ Severe (27-34) } \\
\hline \multicolumn{7}{|l|}{ Extremely severe (35-42) } \\
\hline 2. Anxiety (0-42) & $14.61 \pm 10.68$ & $10.29 \pm 8.44$ & -3.52 & $<0.001$ & 0.39 & \\
\hline Normal (0-6) & $62(36.5)$ & $123(72.4)$ & & & & \\
\hline \multicolumn{7}{|l|}{ Mild (7-9) } \\
\hline \multicolumn{7}{|l|}{ Moderate (10-14) } \\
\hline \multicolumn{7}{|l|}{ Severe (15-19) } \\
\hline \multicolumn{7}{|l|}{ Extremely severe (20-42) } \\
\hline 3. Depression (0-42) & $15.3 \pm 11.64$ & $11.24 \pm 9.81$ & -3.03 & $<0.002$ & 0.80 & \\
\hline Normal (0-9) & $24(14.1)$ & $116(68.2)$ & & & & \\
\hline \multicolumn{7}{|l|}{ Mild (10-12) } \\
\hline \multicolumn{7}{|l|}{ Moderate (13-20) } \\
\hline \multicolumn{7}{|l|}{ Severe (21-27) } \\
\hline Extremely severe (28-42) & & & & & & \\
\hline
\end{tabular}

SLE - systemic lupus erythematosus; FSFI - Female Sexual Function Index; DASS - Depression, Anxiety, and Stress Scales; ANCOVA - analysis of covariance.

from the disease exacerbated their sexual dysfunction. According to some studies, anxiety and depression are more common in SLE patients than the healthy population, and psychological problems have a significant correlation with the socioeconomic status, disease state, and quality of life of SLE patients [23]. Similarly, the results of a study by Meszaros et al. [24] showed that depression, anxiety, and socioeconomic status had a significant correlation with SLE.

In our study, $85.9 \%$ of women with SLE had sexual dysfunction and the effect of SLE on desire, arousal, lubrication, and satisfaction was significant. However, Stein et al. [25] reported that $10 \%$ and $4 \%$ of SLE patients had mild and severe sexual problems, respectively. In a study by Liang et al. [26] 16\% of SLE patients reported sexual difficulties, including more vaginal discomfort and pain during a sexual relationship and difficulties in penetration due to vaginal stiffness. The prevalence of sexual dysfunction was $45.9 \%$ in a study by Morales et al. [7].

Consistent with our results, Curry et al. [18] reported that SLE patients had a higher rate of abstention and depression and a lower frequency of sexual activity, sexual adjustment, and lubrication. According to a study by Shen et al. [27] depression is the major reason for the decreased quality of life of SLE patients and a major factor affecting their sexual problems. 

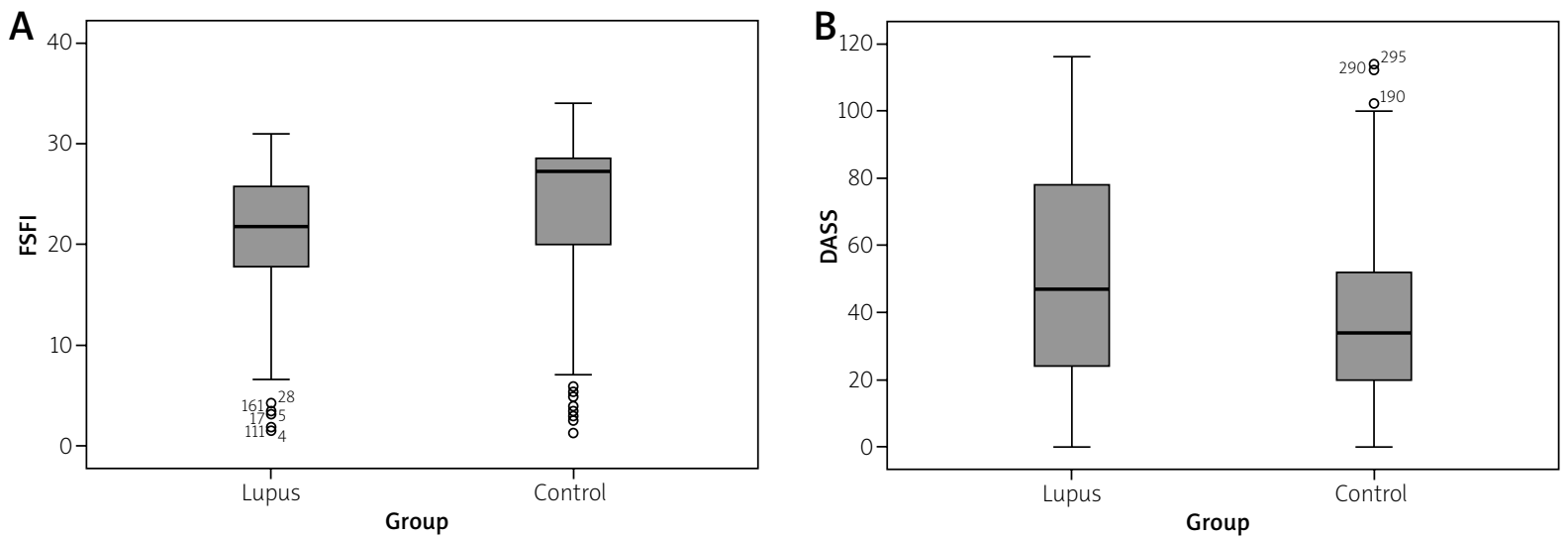

Fig. 1. Female Sexual Function Index and Depression, Anxiety, and Stress Scales changes in two groups.

Table III. Relationships between sociodemographic variables, disease parameters, stress, anxiety, depression, and sexual problems in systemic lupus erythematosus patients

\begin{tabular}{|lcccc|}
\hline \multirow{2}{*}{ Characteristic } & \multicolumn{2}{c}{$r$} & \multicolumn{2}{c|}{ ANCOVA } \\
\cline { 2 - 5 } & $\rho$ & $p$ & $F$ & $p$ \\
\hline Age & -0.41 & $<0.001$ & 0.48 & 0.48 \\
\hline Life status & -0.21 & 0.004 & 3.46 & 0.06 \\
\hline Educational level & 0.017 & 0.82 & 0.68 & 0.17 \\
\hline Job & 0.014 & 0.82 & 0.79 & 0.06 \\
\hline Child number & $-0.201^{\star}$ & 0.009 & 4.29 & 0.03 \\
\hline Child age & $-0.37^{\star *}$ & $<0.001$ & 1.43 & 0.23 \\
\hline Economic status & $0.11^{\star \star}$ & 0.06 & 0.79 & 0.37 \\
\hline Menstrual status & $-0.31^{\star \star}$ & $<0.001$ & 0.003 & 0.96 \\
\hline Marriage duration & -0.41 & $<0.001$ & 2.11 & 0.14 \\
\hline Diagnostic age & $-0.16^{\star}$ & 0.03 & 1.40 & 0.23 \\
\hline Disease duration & $-0.32^{\star *}$ & $<0.001$ & 4.15 & 0.04 \\
\hline SLEDAl & -0.198 & $<0.001$ & 0.09 & 0.75 \\
\hline DASS & $-0.46^{\star \star}$ & $<0.001$ & 3.85 & 0.051 \\
\hline Stress & -0.42 & $<0.001$ & 0.006 & 0.93 \\
\hline Anxiety & -0.40 & $<0.001$ & 7.97 & 0.005 \\
\hline Depression & -0.44 & $<0.001$ & 1.76 & 0.18 \\
\hline
\end{tabular}

SLEDAI - Systemic Lupus Erythematosus Disease Activity Index; DASS - Depression, Anxiety, and Stress Scales; ANCOVA - analysis of covariance; ${ }^{*} p<0.05 ;{ }^{* *} p<0.01$.

We found depression in $85.9 \%$ of our patients, which is consistent with the results of a study by Bachen et al. [8], who reported higher rates of depression and anxiety in female SLE patients, especially young women.

In our study, the results of stepwise regression analysis showed that sexual performance of women with SLE correlated with age, disease activity, depression, and life status. According to the study by Shen et al. [10], stress and anxiety worsen sexual disorders through disturbing social life and activity, which can be improved by counseling [10, 28-30]. The relationship between physical performance and psychological factors may play an important role in sexual and overall health of SLE patients.

Due to cultural considerations regarding sexual matters in Iran and since SLE affects women 9-10 times more than men, only married women were included in this research. Since this study was conducted in Shariati Hospital affiliated with Tehran University of Medical Sciences, the single-center nature of the study might limit its generalizability. Because corticosteroid therapy in SLE patients might have little impact on sexual and mental disorders, we tried to include women with minimal use of corticosteroids and cytotoxic drugs within the last month. Moreover, accurate clinical guidelines should be used to evaluate the direct effect of psychological factors on sexual activity.

\section{Conclusions}

This research was the first study of the sexual and psychological problems of women suffering from SLE in Iran. The results of this study not only can improve the health outcomes of SLE patients but can also decrease their treatment costs. Considering the viewpoint of SLE patients that "physicians only see the disease and our body and do not pay attention to our soul and what we need", our findings may open new horizons in the care and treatment of SLE patients. To achieve this goal, an interdisciplinary approach is required to shift the care of these patients from a biomedical model to a biopsychosocial model [10]. Moreover, increased knowledge and awareness of rheumatologists, nurses, and care providers about physical, sexual, and psychological aspects of care plays an important role in improved management of these patients. 
Table IV. Stepwise regression analyses of sociodemographic variables, stress, anxiety, depression, and their relationship with sexual functions in systemic lupus erythematosus patients

\begin{tabular}{|lcccc|}
\hline Characteristic & Coefficient & Std. error & $t$ & $p$ \\
\hline Age & -0.275 & 0.05 & -5.45 & $<0.001$ \\
\hline Life status & -3.67 & 1.15 & -3.19 & $<0.001$ \\
\hline SLEDAl & -2.95 & 0.62 & -4.75 & 0.001 \\
\hline Child number & 1.58 & 1.85 & 0.87 & 0.38 \\
\hline Child age & 0.08 & 0.09 & 0.89 & 0.37 \\
\hline Economic status & 0.34 & 0.85 & 0.40 & 0.68 \\
\hline Menstrual status & -1.22 & 1.11 & -1.10 & 0.27 \\
\hline Marriage duration & -0.16 & 0.10 & -1.53 & 0.12 \\
\hline Diagnostic age & 0.09 & 0.19 & 0.47 & 0.63 \\
\hline Disease duration & 0.025 & 0.19 & 0.12 & 0.89 \\
\hline DASS & 0.08 & 0.23 & 0.38 & 0.70 \\
\hline Stress & -0.20 & 0.24 & -0.84 & 0.39 \\
\hline Anxiety & -0.10 & 0.24 & -0.41 & 0.67 \\
\hline Depression & -0.14 & 0.03 & -4.09 & $<0.001$ \\
\hline
\end{tabular}

SLEDAI - Systemic Lupus Erythematosus Disease Activity Index; DASS - Depression, Anxiety, and Stress Scales.

Funding opportunities: Tehran University of Medical Sciences, Tehran, Iran.

The authors declare no conflict of interest.

\section{References}

1. Centers for Disease Control and Prevention (CDC). Systemic lupus erythematosus. 2015. Retrieved from: www.cdc.gov/arthritis/basics/lupus.htm.

2. Lau C, Yin G, Mok M. Ethnic and geographical differences in systemic lupus erythematosus: an overview. Lupus 2006; 15: 715-719.

3. Davatchi F, Jamshidi AR, Tehrani Banihashemi A, et al. WHOILAR COPCORD study (stage 1, urban study) in Iran. J Rheumatol 2008; 35: 1384-1390.

4. Jones A, Giles I. Fertility and pregnancy in systemic lupus erythematosus. Indian J Rheumatol 2016; 11: 128-134.

5. Akbarian M, Faezi ST, Gharibdoost F, Shahram F. Systemic lupus erythematosus in Iran: a study of 2280 patients over 33 years. Int J Rheum Dis 2010; 13: 374-379.

6. Kartoz CR. Reproductive Health Concerns in Women with Systemic Lupus Erythematosus. MCN: The American Journal of Maternal/Child Nursing 2015; 40: 220-226.

7. Morales MG, Rubio JC, Peralta-Ramírez M, et al. Impaired sexual function in women with systemic lupus erythematosus: a cross-sectional study. Lupus 2013; 22: 987-995.

8. Bachen EA, Chesney MA, Criswell LA. Prevalence of mood and anxiety disorders in women with systemic lupus erythematosus. Arthritis Care Res 2009; 61: 822-829.

9. Quinzanos I, Davis L, Keniston A, et al. Application and feasibility of systemic lupus erythematosus reproductive health care quality indicators at a public urban rheumatology clinic. Lupus 2015; 24: 203-209.
10. Shen B, He Y, Chen H, et al. Body Image Disturbances Have Impact on the Sexual Problems in Chinese Systemic Lupus Erythematosus Patients. J Immunol Res 2015; Article ID 204513.

11. Knight A, Vickery M, Fiks A, Barg FK.The illness experience of youth with lupus/mixed connective tissue disease: a mixed methods analysis of patient and parent perspectives. Lupus 2016; 25: 1028-1039.

12. McElhone K, Abbott J, Gray J, et al. Patient perspective of systemic lupus erythematosus in relation to health-related quality of life concepts. a qualitative study. Lupus 2010; 19: 1640-1647.

13. World Health Organization. Sexual and reproductive health beyond 2014: Equality, Quality of care and Accountability: position paper 2014.

14. Mohammadi K, Heidari M, Faqihzadeh S. The validation of female sexual function index (FSFI) in the women: Persian Version. PayeshJournal 2008; 7: 270-278.

15. Henry JD, Crawford JR. The short-form version of the Depression Anxiety Stress Scales (DASS-21): Construct validity and normative data in a large non-clinical sample. Br J Clin Psychol 2005; 44: 227-239.

16. Saadat M, Behboodi ZM, Saadat E. Comparison of depression, anxiety, stress, and related factors among women and men with human immunodeficiency virus infection. J Hum Reprod Sci 2015; 8: 48-51.

17. Ho TM, Fernandes M. Patient's sexual health: Do we care enough? J Ren Care 2006; 32: 183-186.

18. Curry SL, Levine SB, Corty E, et al. The impact of systemic lupus erythematosus on women's sexual functioning. I Rheumatol 1994; 21: 2254-2260.

19. Elham R, Moghadam ZB, Hamid H. The effect of sleep health behavioral education on the depression of pregnant women 
with sleep disorders: A randomized control trial. Iran Red Crescent Med J 2015; 17: e11420.

20. Øtensen M. New insights into sexual functioning and fertility in rheumatic diseases. Best Pract Res Clin Rheumatol 2004; 18: 219-232.

21. Zahra BM, Elham R. The Prevalence of Depression in Pregnant Women with Sleep Disorder. J Psychiatry 2014; 17: 157.

22. Panush RS, Mihailescu GD, Gornisiewicz MT, et al. Sex and arthritis. Bull Rheum Dis 2000; 49: 1-4.

23. Shen B, Tang W, Feng G, et al. The Correlations of Disease Activity, Socioeconomic Status, Quality of Life, and Depression/Anxiety in Chinese Patients with Systemic Lupus Erythematosus. Clin Dev Immunol 2013; 2013: 270878.

24. Meszaros ZS, Perl A, Faraone SV. Psychiatric symptoms in systemic lupus erythematosus: a systematic review. J Clin Psychiatry 2012; 73: 993-1001.

25. Stein H, Walters K, Dillon A, et al. Systemic lupus erythematosus - a medical and social proile. J Rheumatol 1986; 13 : $570-576$
26. Liang $M H$, Rogers $M$, Larson $M$. The psychosocial impact of systemic lupus erythematosus and rheumatoid arthritis. Arthritis Rheum 1984; 27: 13-19.

27. Shen B, Feng G, Tang W, et al. The quality of life in Chinese patients with systemic lupus erythematosus is associated with disease activity and psychiatric disorders: a path analysis. Clin Exp Rheumatol 2014; 23: 101-107.

28. Fatemeh Y, Zahra BM, Soror $P$, et al. Sexual and reproductive health problems of female university students in Iran: a qualitative study. Global Journal of Health Science 2015; 7: 278-285.

29. Zahra BM, Elham R, Fariba KY, et al.The effect of sexual health education program on women sexual function in Iran. JRHS 2015; 15: 124-128.

30. Khakbazan Z, Daneshfar F, Behboodi-Moghadam Z, et al. The effectiveness of the Permission, Limited Information, Specific suggestions, Intensive Therapy (PLISSIT) model based sexual counseling on the sexual function of women with Multiple Sclerosis who are sexually active. Mult Scler Relat Disord 2016; 8: 113-119. 